Arch. Tierz, Dummerstorf 44 (2001) 1, 15-22

Aus der West-Ungarischen-Universitat, Mosonmagyarovar, Ungarn

LASZLO GULYAS Und JANOS IVÁNCSICS

\title{
Zusammenhänge zwischen der somatischen Zellzahl und einigen eutermorphologischen Eigenschaften
}

\begin{abstract}
Summary
Title of the paper: Relationship between the somatic cell count and certain udder-morphologic traits The authors carried out their udder-morphologic (the shape, hanging (up) and depth of udder; the length and diameter of teats and the space between them; the length of ductus papillaris) research in a high blood-share black-spotted Holstein-Friesian $\left(\mathrm{R}_{3}-\mathrm{R}_{4}\right)$ cow population in a West Hungarian large dairy farm with a stock of 500 cows.

The results of examinations carried out by groups offspring draw the attention to the close correlation ( $r=0.62$ 0.88 ) between certain udder-morphologic traits and the pigmentation of teats as well as between the values of somatic cell count.

Furthermore, the authors observed that there is also a close correlation $(r=-0.58-\quad-0.89$ between the length of teat duct (ductus papillaris) and the values of somatic cell count.

All biological characteristics (udder-morphologic, pigmentation of teats, length of ductus papillaris) together with those influencing somatic cell count are to be taken into account in the selection work having the aim of decreasing somatic cell count.
\end{abstract}

Key Words: somatic cell count, udder-morphologic traits, ductus papillaris, dairy caitle

\section{Zusammenfassung}

An 406 Trchtern von 5 Bullen wurden Zusammenhänge zwischen einigen morphologischen Eutereigenschaften und der somatischen Zellzahl untersucht. In einem 500 Kuhe umfassenden Bestand aus $R_{3}-R_{4}$ Tieren der Rassen Ungarisches Fleckvieh x Holstein-Friesian wurden die Merkmale: Euterform, Euteransatz, Eutertiefe, Zitzenabstand, Zitzenlănge, Zitzendurchmesser, Zitzenpigmentierung, Länge des Zitzenkanals und die somatische Zellzahl untersucht. Enge positive Korrelationen konnten zwischen Euterform, Euteransatz und Eulertiefe zur Zellzahl nachgewiesen werden. Negative Beziehungen ergaben sich zwischen der Lange des Zitzenkanals und der somatischen Zellzahl. Es werden die Selektionsmöglichkeiten zur Reduzierung der Zellzahl diskutiert.

Schlosselwörter: Somatische Zellzahl, morphologische Eutermerkmale, Zitzenkanal, Milchrind

\section{Einführung}

Die somatische Zellzahl ist ein für die Diagnostizierung und Messung einer Euterentzündung anwendbares Merkmal. Eine Euterentzündung ist eine krankhafte Veränderung des Euters, die mit Sekretionsstörungen, mit einer von der Norm abweichenden Milchzusammensetzung und einer Verminderung der Milchleistung einhergeht. Euterentzlindungen und eine hohe somatische Zellzahl haben viele Ursachen. Sehr wichtig ist es, die Grunde ihrer Entstehung zu kennen und mögliche Ursachen abzustellen Eine Erhöhung des Milchleistungsniveaus mit den Mitteln der Züchtung hat vor allem Bestände mit höheren Milchleistungen bezüglich der Resistenz des Euters negativ beeinflusst (DOHY, 1985). Dies ist aber kein gesetzmäßiger Zusammenhang, sondern er kann genetisch bedingt sein und darlber hinaus mit fehlender Harmonie zwischen 
Umwelt- und Managementbedingungen erklärt werden (TỖRÖS, 1980). Während auf die Verbesserung der Leistungsergebnisse nicht verzichten kann, müssen komplexe Bemühungen gegen die Mastitis ein wichtiger Teil der Züchtungsarbeit bleiben (DOHY, 1985, 1999; HORVÁTH, 1990; IVÁNCSICS, 1999). Einer der wichtigsten Aspekte zur Vorbeugung gegen eine Euterentzündung ist eine auf die Verbesserung der Euterentwicklung gerichtete Selektion (MONARDES et al., 1999, BROTHERSTONE et al., 2000).

Die Resistenz gegen die Mastitis ist eine komplexe Eigenschaft, deren Vererbbarkeit mit Hilfe mehrerer Merkmale untersucht werden kann. So werden in einigen Quellen für die Mastitis $\mathrm{h}^{2}$-Werte zwischen 0,05 bis 0,50 angegeben. Diese relativ niedrigen Werte reichen dennoch aus um bei großer Varianz mittels züchterischer Maßnahmen eine Verbesserung der Resistenz anzustreben (MALMBERG, 1992; KALM, 1993). Obwohl die äußere Bewertung des Euters unumgänglich ist und eine relativ sichere Auskunft über die morphologischen Merkmale des Euters gibt, kann auf genaue Messungen beruhende Untersuchungen nicht verzichtet werden. Für morphologische und strukturelle Eigenschaften des Euters werden $h^{2}$-Werte von 0,16 bis 0,37 angegeben (BROTHERSTONE et al., 1990). Damit könnten diese Merkmale schon in wenigen Generationen verbessert werden.

GROOTENHUIS (1980) weist darauf hin, dass bei einer auf die Zellzahlsenkung gerichteten Selektion die Rassen am vielversprechendsten sind, bei denen die durchschnittliche Zellzahl ein heterogeneres Bild bei den weiblichen Nachkommen aufweist, also die Varianz dieser Eigenschaft größer ist. DOHY (1985) fand einen positiven Zusammenhang zwischen der besseren Persistenz und der geringeren Mastitisanfälligkeit.

Schon bei der Auswahl der Färsen ist es wichtig, neben der zu erwartenden Milchleistung die Mastitisanfälligkeit der Vorfahren und den anatomischen Euteraufbau zu kennen (HAMANN und REICHMUT, 1989; TAKÁTSY, 1991).

Für eine Zuchtwertschätzung der Mastitisresistenz benötigt man die Laktationsdaten von minimal 80, nach Meinung anderer Autoren von 200 Kühen in der ersten Laktationsperiode (DOHY, 1989; MALMBERG, 1992; ERIKSSON und SOLBU, 1993; KALM, 1993). Die Auswertung und die Selektion müssen auf eine gezielte Anpaarung der Bullenmütter und deren Familien erweitert werden (GROOTENHUIS, 1980; Mc DANIEL, 1986). Auch HÁMORI (1980) fand, dass über die Untersuchung der Väter hinaus, die Kuhfamilien in die Untersuchungen einbezogen werden sollten. Daher sind Kuhfamilien in denen gehäuft Tiere wegen Mastitis früh gemerzt wurden, von der Bullenauswahl auszuschließen.

Das Ziel der Untersuchungen bestand in der Bewertung verschiedener Eutermerkmale einzelner Bullennachkommenschaften sowie der Prüfung von Zusammenhängen zwischen diesen und der somatischen Zellzahl.

\section{Material und Methode}

Die Untersuchungen wurden in der 500 Tiere fassenden Rinderzuchtanlage der "Kisalföld" MGSz. in Bogyoszló/Ungarn durchgeführt.

Bei dem Bestand handelt es sich um $R_{3}$ bzw. $R_{4}$ Tiere des Genotyps Ungarisches Fleckvieh x Schwarzbunt Holstein-Friesian. Die Haltung der Tiere erfolgt im Laufstall 
mit Tiefstreu. Gemolken wurde täglich zweimal in einem Fischgrätenmelkstand mit 2 $x \quad 2 \times 10$ Ständen. Die Durchschnittsmilchleistung der Herde betrug im Untersuchungszeitraum 7,264 kg/Jahr je Kuh. Die in die Untersuchung einbezogenen Kühe befanden sich in der 2. bis 4. Laktation. Die Messungen erfolgten zwischen dem 60. und 120. Laktationstag.

Folgende Merkmale wurden einbezogen

- Die morphologische Untersuchung des Euters (Form, Ansatz, Tiefe)

- Die morphologische Untersuchung der Zitzen (Abstand, Länge, Durchmesser)

- Die Pigmentierung der Zitzen (voll, teils, lückenhaft)

- Die Länge des Zitzenkanals (ductus papillaris)

- Die Hohe der somatischen Zellzahl der Milch

Es wurde der Zusammenhang zwischen den oben genannten Aspekten und der somatischen Zellzahl der Milch bei 406 Nachkommen von 5 Bullen statistisch ausgewertet. Berechnet wurden Mittelwert, Streuung und Korrelationskoeffizienten $(p<0,01)$

Die Analyse der Milchproben erfolgte in der Állattenyésztési Teljesítményvizsgáló Kft., Gödöllő (Gesellschaft für Leistungsprüfung der Tierzucht). Die Eutermorphologie wurde mit einer Punktezahl ( 1 bis $5=$ beste) bewertet. Die Messungen der Länge des Zitzenkanals (ductus papillaris) wurden an jeweils 18 bis 20 gleich alten Töchtern von fünf Bullen durchgeführt. Messungen der Länge des Zitzenkanals wurden mit einem selbst entwickelten Gerät vorgenommen (Abb.). Für die Messung wird der Messfühler in den Zitzenkanal eingeführt und dann langsam soweit zurückgezogen, bis das am Ende des Fühlers befindliche Kugelchen die innere Begrenzung des Kanals erreicht. Nach dem Heranschieben der Hülse des Messgerätes an die Zitzenspitze, kann der Messwert direkt abgelesen werden.

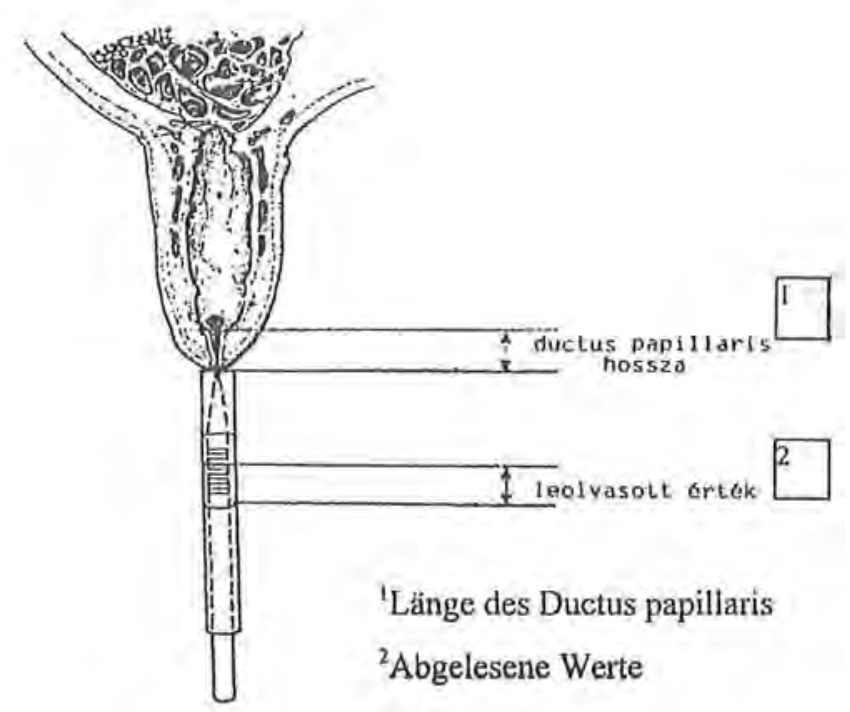

Abb.: Messung der Lange des Zitzenkanals (ductus papillaris) (Measuring of Length of Ductus papillaris) 
Ergebnisse und Diskussion

Zwischen den einzelnen Bullennachkommenschaften ergaben sich erhebliche Differenzen in den morphologischen Eutereigenschaften (Tab. 1), Die Zitzenabstände lassen für einzelne Bullennachkommenschaften teilweise sehr unterschiedliche Euterproportionen erkennen. Bei den phänotypischen Korrelationskoeffizienten einzelner Eutermerkmale zur somatischen Zellzahl bestanden zur Euterentwicklung $(\mathrm{r}=0,64-$ $0,83)$, dem Euteransatz $(r=0,62-0,84)$ und dem Bodenabstand $(r=0,67-0,88)$ enge positive Beziehungen. Niedrigere Korrelationskoeffizienten wurden dagegen für die Merkmale Zitzenabstand, Länge und Durchmesser der Zitzen geschätzt.

Tabelle I

Eutermorphologische Untersuchungen von Bullen-Nachkommengruppen (Udder morphological examination of bull-offsprings groups)

\begin{tabular}{|c|c|c|c|c|c|c|}
\hline Merkmal & Namen der Bullen & $\begin{array}{c}7585 \\
\text { ANTON }\end{array}$ & $\begin{array}{l}10151 \\
\text { ELERT }\end{array}$ & $\begin{array}{c}9414 \\
\text { CZIMI }\end{array}$ & $\begin{array}{l}11845 \\
\text { VEIT }\end{array}$ & $\begin{array}{l}10566 \\
\text { ERMES }\end{array}$ \\
\hline Zahl der Nachkommen & $n$ & 18 & 85 & 67 & 165 & 71 \\
\hline Euterform (Punkte) & $\begin{array}{l}\text { Durchschnitt } \\
\text { Variation } \\
\text { CV\% }\end{array}$ & $\begin{array}{c}3,39 \\
0,85 \\
25,08\end{array}$ & $\begin{array}{c}4,11 \\
0,46 \\
11,18\end{array}$ & $\begin{array}{c}3,15 \\
0,73 \\
23,06\end{array}$ & $\begin{array}{c}4,10 \\
0,64 \\
15,63\end{array}$ & $\begin{array}{c}4,10 \\
0,79 \\
19,22\end{array}$ \\
\hline Euteransatz (Punkte) & $\begin{array}{l}\text { Durchschnitt } \\
\text { Variation } \\
\text { CV\% } \\
\end{array}$ & $\begin{array}{c}3,17 \\
0,62 \\
19,53\end{array}$ & $\begin{array}{c}4,16 \\
0,69 \\
16,55 \\
\end{array}$ & $\begin{array}{r}3,30 \\
0,84 \\
25,53 \\
\end{array}$ & $\begin{array}{c}4,15 \\
0,59 \\
14,15 \\
\end{array}$ & $\begin{array}{c}4,15 \\
0,88 \\
21,09\end{array}$ \\
\hline Eutertiefe $(\mathrm{cm})$ & $\begin{array}{l}\text { Durchschnitt } \\
\text { Variation } \\
\text { CV\% } \\
\end{array}$ & $\begin{array}{c}44,00 \\
4,21 \\
9,58 \\
\end{array}$ & $\begin{array}{l}47,00 \\
2,18 \\
4,60 \\
\end{array}$ & $\begin{array}{c}42,85 \\
4,56 \\
10,65 \\
\end{array}$ & $\begin{array}{c}46,30 \\
3,57 \\
7,71 \\
\end{array}$ & $\begin{array}{c}46,95 \\
4,85 \\
10,33 \\
\end{array}$ \\
\hline $\begin{array}{l}\text { Abstand zwischen den } \\
\text { linken vorderen und den } \\
\text { rechten hinteren Zitzen }\end{array}$ & $\begin{array}{l}\text { Durchschnitt } \\
\text { Variation } \\
\text { CV\% }\end{array}$ & $\begin{array}{l}17,06 \\
2,44 \\
14,29\end{array}$ & $\begin{array}{l}20,63 \\
2,75 \\
13,34 \\
\end{array}$ & $\begin{array}{c}17,30 \\
3,36 \\
19,44 \\
\end{array}$ & $\begin{array}{c}20,95 \\
2,86 \\
13,63 \\
\end{array}$ & $\begin{array}{l}18,20 \\
2,67 \\
14,66\end{array}$ \\
\hline $\begin{array}{l}\text { Abstand zwischen den } \\
\text { linken hinteren und den } \\
\text { rechten vorderen Zitzen } \\
(\mathrm{cm})\end{array}$ & $\begin{array}{l}\text { Durchschnitt } \\
\text { Variation } \\
\text { CV\% }\end{array}$ & $\begin{array}{c}11,89 \\
3,36 \\
28,25\end{array}$ & $\begin{array}{c}16,32 \\
2,85 \\
17,46\end{array}$ & $\begin{array}{l}11,10 \\
2,86 \\
25,78\end{array}$ & $\begin{array}{c}16,00 \\
2,51 \\
15,71\end{array}$ & $\begin{array}{l}14,10 \\
2,38 \\
16,89\end{array}$ \\
\hline $\begin{array}{l}\text { Abstand zwischen den } \\
\text { linken vorderen und linken } \\
\text { hinteren Zitzen }(\mathrm{cm})\end{array}$ & $\begin{array}{l}\text { Durchschnitt } \\
\text { Variation } \\
\text { CV\% }\end{array}$ & $\begin{array}{l}13,28 \\
2,52 \\
18,98\end{array}$ & $\begin{array}{l}17,26 \\
2,33 \\
13,49\end{array}$ & $\begin{array}{l}13,50 \\
2,60 \\
19,25\end{array}$ & $\begin{array}{c}18,40 \\
2,21 \\
12,01\end{array}$ & $\begin{array}{l}16,20 \\
2,04 \\
12,60\end{array}$ \\
\hline Zitzenlänge (cm) & $\begin{array}{l}\text { Durchschnitt } \\
\text { Variation } \\
\text { CV\% }\end{array}$ & $\begin{array}{l}6,83 \\
0,84 \\
12,30 \\
\end{array}$ & $\begin{array}{l}8,34 \\
0,63 \\
7,49\end{array}$ & $\begin{array}{l}6,63 \\
0,57 \\
8,58\end{array}$ & $\begin{array}{l}8,13 \\
0,78 \\
9,55\end{array}$ & $\begin{array}{c}7,25 \\
0,89 \\
12,36 \\
\end{array}$ \\
\hline $\begin{array}{l}\text { Durchmesser der Zitzen } \\
(\mathrm{mm})\end{array}$ & $\begin{array}{l}\text { Durchschnitt } \\
\text { Variation } \\
\text { CV\% }\end{array}$ & $\begin{array}{c}25,17 \\
1,25 \\
4,96\end{array}$ & $\begin{array}{c}26,42 \\
1,07 \\
4,05\end{array}$ & $\begin{array}{c}24,50 \\
1,28 \\
5,24\end{array}$ & $\begin{array}{c}26,35 \\
1,46 \\
5,54\end{array}$ & $\begin{array}{c}24,70 \\
1,59 \\
6,45\end{array}$ \\
\hline
\end{tabular}

Der Abstand zwischen den Zitzen und der Euterabstand vom Boden sind vererbbar und zeigen eine negative genetische Korrelation zu Euterentzündungen (MADSEN et al., 1987; BROTHERSTONE et al, 1990). Nach RYNIEWICZ (1980) sind Kühe mit mangelhaften eutermorphologischen Eigenschaften empfindlicher gegen eine Euterentzündung. Besonders bei Kühen mit trichterförmigen Zitzenenden wurde die Krankheit öfter beobachtet. MONARDES et al. (1990) haben festgestellt, dass es zwischen einigen äußeren Eigenschaften wie dem Zusammentreffen von Eutersitz, den Proportionen der vorderen und hinteren Euterhälften, der Gewebestruktur, der Stellung 
der vorderen und hinteren Zitzen und der durchschnittlichen somatischen Zellzahl während der Laktation, eine verhältnismäßig enge Beziehung gibt. So kann eine erwünschte hohe Punktzahl der Euterbewertung mit einer niedrigen Zellzahl verbunden sein.

Die Milchleistung und die Melkgeschwindigkeit hängen mit dem Eutertyp und der Form der Zitzen zusammen. SowohI die Strichkanallänge als auch die Zitzenwanddicke nehmen Einfluß auf den Verlauf der Milchabgabe. Mit einem Anstieg beider Eigenschaften konnten sowohl eine Abnahme des höchsten Milchflusses als auch des durchschnittlichen Minutengemelks (DGM) beobachtet werden (NAUMANN und FAHR, 2000).

Kühe mit einem wannenforrmigen Euter und mit zylinderförmigen Zitzen zeigen eine bessere Milchleistung und sind besser melkbar (SINGH et al., 1997).

SOBAR et al. (1994) haben festgestellt, dass Kühe mit besserer Melkbarkeit eine Milch mit signifikant geringerer somatischer Zellzahl produzieren als schlecht melkbare Tiere. Die schwierigere Melkbarkeit und die damit verbundene langsame Milchabgabe hängen häufig signifikant mit dem Auftreten der Mastitis zusammen. Auch SOMOS (1987) fand einen relativ engen Zusammenhang zwischen der Häufigkeit der Mastitis und den Euterproportionen, dem Ansatz des Euters, der Eutertiefe, dem Euterabstand vom Boden und der zusammenfassenden Euterpunkzahl $(r=-0,29$ bis $-0,47)$. Mastitishäufigkeit trifft auch für ungarische Bestände zu, wenn die Zitzen länger als $6,5 \mathrm{~cm}$ und breiter als $2,5 \mathrm{~cm}$ sind. Sie sind eher verletzbar, besonders wenn sie eine von der Norm abweichende Form haben. Weniger gefährdet sind dagegen Zitzen mit einer leichten Kegelform, abgerundeten Enden und einer runden, punktförmigen Öffnung (McDANIEL, 1986). Bei der Zitzenbeurteilung ist zu beachten, dass die Vererbung der Zitzenentwicklung nur an solchen Tieren sicher auswertbar ist, die noch nicht an Mastitis erkrankt waren, da die äußeren Mängel auch die Folgen früherer Gesundheitsschäden des Euters und falscher Melkmethoden sein können (KATONA, 1991). IVANCSICS et al. (1996), GULYAS et al. (1998), IVÁNCSICS (1999) und DOHY (1999) betonen den Zusammenhang zwischen der Pigmentierung der Zitzen und der somatischen Zellzahl.

Tabelle 2 zeigt am vorliegenden Material das Auftreten von Zitzenpigmentierungen sowie die somatische Zellzahl der untersuchten Bullennachkommenschaften.

Sowohl bei der somatischen Zellzahl als auch der Zitzenpigmentierung ergaben sich signifikante Unterschiede zwischen den Nachkommengruppen $(\mathrm{P}<0,01)$. Die durchschnittlichen Zellzahlen der einzelnen Bullennachzuchten, geprüft im gleichen Bestand, wiesen deutliche Unterschiede auf. Der Anteil an Tieren in den Nachzuchtgruppen die eine somatische Zellzahl von über $400 \times 10^{3} / \mathrm{ml}$ aufwiesen, erreichte Werte zwischen $11,1 \%$ und $31,8 \%$.

Die Untersuchung der Länge des Zitzenkanals (ductus papillaris) wurde an jeweils 18 bis 20 gleich alten Bullentöchtern vorgenommen (Tab. 3). Es zeigten sich zwischen den Nachkommenschaften erhebliche Unterschiede. Die Gruppen der Väter „Elert, Veit und Ermes" hatten die größte Länge des Zitzenkanals in Verbindung mit einer günstigen, relativ niedrigen Zellzahl.

Bei den Nachkommen der Bullen mit offenbar geöffnetem und kürzerem Schließmuskel („Anton und Czimi") konnten wesentlich höhere somatische Zellzahlen festgestellt 
Tabelle 2

Zitzenpigmentation und die somatische Zellzahl der Bullen-Nachkommengruppen (Udder pigmentation and somatic cell count of bull's offspring groups)

\begin{tabular}{|c|c|c|c|c|c|}
\hline $\begin{array}{l}\text { Namen der Bullen } \\
\text { Merkmal }\end{array}$ & $\begin{array}{c}7585 \\
\text { ANTON } \\
\end{array}$ & $\begin{array}{l}10151 \\
\text { ELERT }\end{array}$ & $\begin{array}{l}9414 \\
\text { CZIMI }\end{array}$ & $\begin{array}{l}11845 \\
\text { VEIT } \\
\end{array}$ & $\begin{array}{l}10566 \\
\text { ÉRMES }\end{array}$ \\
\hline $\begin{array}{l}\text { Zitzen ohne } \\
\text { Pigmentierung } \\
\text { a Tiere }(\mathrm{n}) \\
=\text { Håufigkeit }(\%) \\
\quad \text { Durchschnitt, somatische } \\
\quad \text { Zellzahl }\left(10^{3} / \mathrm{ml}\right) \\
\end{array}$ & $\begin{array}{c}14 \\
77,77 \\
489\end{array}$ & $\begin{array}{c}21 \\
24,70 \\
362\end{array}$ & $\begin{array}{c}41 \\
61,20 \\
547\end{array}$ & $\begin{array}{c}33 \\
20,00 \\
209\end{array}$ & $\begin{array}{c}24 \\
33,80 \\
204\end{array}$ \\
\hline $\begin{aligned} \text { Teilweise. Pigmentierung } \\
\text { "Tiere }(\mathrm{n}) \\
\text { " Haufigkeit }(\%) \\
\text { Durchschnitt, somatische } \\
\text { Zellzahl }\left(10^{3} / \mathrm{ml}\right) \\
\end{aligned}$ & $\begin{array}{c}4 \\
22,23 \\
318\end{array}$ & $\begin{array}{c}36 \\
42,35 \\
288\end{array}$ & $\begin{array}{c}23 \\
34,32 \\
438\end{array}$ & $\begin{array}{c}73 \\
44,24 \\
147\end{array}$ & $\begin{array}{c}28 \\
39,44 \\
149\end{array}$ \\
\hline $\begin{aligned} \text { Voll pigmentierte Zitzen } \\
\text { - Tiere }(\mathrm{n}) \\
. \quad \text { Haufigkeit }(\%) \\
\text { - Durchschnit, somatische } \\
\text { Zellzahl }\left(10^{3} / \mathrm{ml}\right) \\
\end{aligned}$ & - & $\begin{array}{c}28 \\
32,95 \\
187\end{array}$ & $\begin{array}{c}3 \\
4,48 \\
312\end{array}$ & $\begin{array}{c}59 \\
35,76 \\
112\end{array}$ & $\begin{array}{c}19 \\
26,76 \\
128\end{array}$ \\
\hline
\end{tabular}

Tabelle 3

Somatische Zellzahl und Lange des ductus papillaris und Korrelationskoeffizienten bei den Nachkommengruppen (Correlation between somatic cell count and ductus papillaris at bulls' offspring groups)

\begin{tabular}{l|c|c|c|c}
\hline Namen der Bullen & $\begin{array}{c}\text { Zahl der } \\
\text { untersuchten } \\
\text { Nachkommen } \\
(\mathrm{n})\end{array}$ & $\begin{array}{c}\text { Durchschnittliche } \\
\text { somatische Zellzahl } \\
\text { bei den } \\
\text { Nachkommen } \\
\left(10^{3} / \mathrm{ml}\right)\end{array}$ & $\begin{array}{c}\text { Länge des } \\
\text { ductus } \\
\text { papillaris } \\
(\mathrm{mm})\end{array}$ & $\begin{array}{c}\text { Korrelation zwischen } \\
\text { dem ductus papillaris } \\
\text { und der somatischen } \\
\text { Zellzahl } \\
(\mathrm{r})\end{array}$ \\
\hline 7585 ANTON & 18 & 451 & 6,89 & $-0,58^{* * *}$ \\
\hline 10151 ELERT & 19 & 177 & 9,89 & $-0,75^{* * *}$ \\
\hline 9414 CZIMI & 20 & 542 & 6,95 & $-0,78^{* * *}$ \\
\hline 11845 VEIT & 20 & 200 & 9,45 & $-0,73^{* * *}$ \\
\hline 10 566 ERMES & 20 & 199 & 8,25 & $-0,89^{* * * *}$ \\
\hline
\end{tabular}

$\because P>0,01$

\section{werden.}

Die signifikanten, negativen Korrelationskoeffizienten zwischen diesen beiden Merkmalen ergaben Werte von $r=-0,58$ bis $-0,89$ und bewiesen den Einfluss der Zitzenmorphologie auf die somatische Zellzahl. In einigen Ländern werden Kühe mit Strafpunkten bewertet deren Zitzenkanal zu kurz ist. LOJDA et al. (1980) fanden einen signifikanten Zusammenhang zwischen trichter- und kraterförmigen Zitzenenden und der Häufigkeit der Euterentzündung. Ein wichtiger Faktor der Mastitisgefährdung ist die unterschiedliche Milchabgabezeit der einzelnen Viertel. Nach TOTH (1983) liegt das durchschnittliche Minutengemelk (DGM) bei $3-5 \mathrm{~kg} / \mathrm{Min}$. Erwünscht sind durchschnittliche Minutengemelke (DGM) von 2,0 bis 4,0 kg/Min, wobei der Anstieg des DGM mit einer höheren Zellkonzentration verbunden ist (KRÄUSSLICH, 1997). Schlussfolgernd aus den vorliegenden Ergebnissen kann festgestellt werden, dass unter 
dem Aspekt einer Verbesserung der Eutergesundheit, gemessen an der somatischen Zellzahl, dieses Merkmal direkt in die Zuchtwertschätzung der Bullen einbezogen werden sollte. Dieses Ergebnis befindet sich in Übereinstimmung mit Literaturaussagen (Mc DANIEL, 1984; DOHY, 1985, 1999; SOMOS, 1987; VÁGI, 1996, 1998; IVÁNCSICS, 1999) und wird in einer Reihe von Ländern bereits erfolgreich praktiziert (BRASKAMP et al., 2000) Die züchterischen Bemühungen zur Verbesserung einzelner eutermorphologischer Merkmale sind zu verstärken.

\section{Literatur}

BRASKAMP, E.W.; BIJMA, P.; GROEN, AB.F.; VOSS, H.; BOVENHUIS, H.; HARLIZIUS, B.; VAN ARENDONK, J.H.M.:

Zuchterische Aspekte der nachhaltigen Rinderproduktion. Arch. Tierz, Dummerstorf 43 (2000) Sonderheft, 34-39

BROTHERSTONE, S. et al.:

Estimation of genetic parameters for linear andmiscellancous typetraits in Holstein-Frisian dairy cattle DOHY, J: Livestock Prod. Science, 26 (1990), 177-192

Genetic possibilities of mastitis control. Tudomány és Mezögazdaság (Science and Agriculture). ^ DOHY, J:; (1985), 24-27

Genetic basis of animal breeding, Mezögazdasági Kiadó (Agricultural Publisher), Budapesı. 303. p.,
1989 DOHY, J:

Genetic problems of udder health. Actual problems of quality in milk production. PATE, Keszthely. 1999

ERIKSSON, J.A.; SOLBU, $\mathrm{H}_{\text {, }}$

Practical experience of breeding for health traits in Scandinavia. $44^{\text {th }}$ Ann. Meeting of the EAAP Aarhus - Denmark. 1993

GROOTENHUIS, G.:

In: BASSALIK, CHABIELSKA, RYNIEWICZ (ed.): Resistant factors and genetic aspects of mastitis control, Proc. Int. Conf., Jablona-Poland, 173-200, p. 1980

HAMANN, J.; REICHMUTH, J,:

Leitlinien zur Bekämpfung der Mastitis des Rindes als Bestandsproblem. DVG, Kiel - Deutschland, 56. p. 1989

GULYÁS, L.; KOVẢCSNÉ GAÁL, K; GULYẢs, T.; BÖJTÖ, J.:

Analysis of the correlation between the somatic cell count content of milk and morphological characteristics of the udder. XXVII. Óvár Scientific Symposium: New challenges in agriculture HẢMORI, D.:

In: BASSALIK-CHABIELSKA, L., RYNIEWICZ, Z. (ed.): Resistant factors and genetic aspects of HORVÁTH, L:: System of requirements towards quality milk production. In: AGOE-Agroinform, Budapest, 71-76. p. 1990

IVȦNCSICS, J.; GULYẢS, L.; DAMJANOVICH, S.; GÁSPAR, R.; KRASZNAI, Z.:

Biological and technological factors of hygienic milk production. XXVI. Óvár Scientific Symposium: New challenges and strategies in agricultural production. Mosonmagyarovár. 1996. Sept. 25. 1. vol. 5355. p.

IVÁNCSICS, J.;

Improvement of milk production in cattle breeding, Academic doctoral thesis, Mosonmagyarovar, IVANCSICS, J.:

Possibilities of improving milk quality. actual problems in milk production. PATE, Keszthely, 1999

KALM, E.:

Improving reproductive-biological capacity and udder health state of cattle using methods of breeding. In: Proc, Conf. on Cattle fertility problems. AOE. Budapest, 5-13. p. 1993 
KATONA, F,:

Aspects of udder health in case of machine milking. Leciure. GATE, post-graduate engineer training course. 1991

KRÄUSSLICH, H.:

In: KRÄUSSLICH und BREM G.: Tierzucht und allgemeine Landwirtschaftslehre für Tiermediziner. Ferdinand Enke Verlag, Stuttgart, 1997

LOJDA, L.; STAVIKOVÁ, M.; ZAKOVÁ, M.: In: BASSALIK-CHABIELSKA, L., RYNIEWICZ, Z, (ed.): Resistant factors and genetic aspects of mastitis control. Proc, Int. Conf., Jablona-Poland, 261-276. p. 1980

LOTTHAMMER, K.-H.; WITTKOWSKI, G.: Fruchtbarkeit und Gesundheit der Rinder, Verlag Eugen Ulmer, Stuttgart, 1994

MADSEN, P.; NIELSEN, S.M.; RASMUSSEN, M.: Investigations on genetic resistance to bovine mastitis. Report from the NIAS, Denmark, 176-185. p. 1987

MALMBERG, G.:

Breeding for healthier cows. Proc. The $71^{\text {th }}$ World Ayrshire Conf., Jönköping - Sweden. 1992

MCDANIEL, B.T:

Progeny testing for disease resistance and stayability, In: Progeny testing methods in dairy cattle, Bulletin of IDF/EEAP Symp., Prague, 173-176. p. 1984

MCDANIEL, B.T.:

A breeding programme for dairy cattle. ÁGOK-Agroinform, Budapest, 22-45. p. 1986

MONARDES, H.G.; CUE, R.I.; HAYES, J.F.:

Correlations between udder conformation traits and somatic cell count in Canadian Holstein cows, J. Dairy Sci., 73 (1990), 1337-1342

NAUMANN, 1.;FAHR, R,-D;: Untersuchungen zum Milchfluss aus Eutervierteln. Arch. Tierz., Dummerstorf 43 (2000) 5, 431-440

RYNIEWICZ, Z: Factors influencing the development of mastits. Állattenyésztés és Takarmányozás (Animal Breeding and Feeding). 43. 6. 529-534. p. 1994

SOMOS, Z:

A correlation between the morphological characteristics of the udder and mastitis. diploma thesis

TÖRÖS, I.: (post/graduate engineer training course), GATE, 38, p. 1987

Our duties in improving the situation of animal health care and reproduction of high-production Tó'TH, L.: capacity stocks. In: breeding Conference for state farms. ÁGOK-Agroinform, 72-74, p.1980

VAGI, J.:

Basic technical and biological aspects of machine milking Holstein Friesian stocks, In: Cattle Breeding Symposium for State dairy farms. AGOK-Agroinform. Budapest, 72-74. p. 1983

Determining a grading system for the somatic cell count of cows using logarithmic transformation for mastitis resistance analyses. XXVI, Óvár Scientific Symposium? New challenges and strategies in VÁGI, J.: agricultural production. Mosonmagyaróvár. 1996. Sept, 25, I. Vol. 199-203, p.

Genetic methodological analyses using the grading system of somatic cell count in dairy cattle stocks. XXVII, Óvár Scientific Symposium: New challenges and strategies in agricultural production. Mosonmagyaróvár. 1996. Sept. 25, I.Vol, 180-184. p. 1998

Eingegangen: 25.05 .2000

Akzeptiert: 23.11 .2000

Anschirift der Verfasser

Ass. Prof. LASZLO gULYAS, Prof. Dr. Dr. h.c. JANOS IVÁNCSICS

West-Ungarische-Universitat

Agrarwissenschaftliche Fakultát

H-9220 MOSONMAGYAROVAR 